\title{
Separation of High Order Harmonics with Fluoride Windows
}

\author{
T. K. Allison, ${ }^{1,2 *}$ J. van Tilborg, ${ }^{2}$ T. W. Wright ${ }^{1,2}$ M. P. Hertlein ${ }^{2}$, R. W. Falcone ${ }^{1,2}$, and A. \\ Belkacem $^{2}$ \\ ${ }^{1}$ Dept. of Physics, University of California, Berkeley \\ 366 LeConte Hall, Berkeley CA, 94720, USA \\ ${ }^{2}$ Lawrence Berkeley National Laboratory, \\ 1 Cyclotron Road, Berkeley CA, 94720 USA \\ *Corresponding author: TKAllison@lbl.gov
}

Compiled October 23, 2008

\begin{abstract}
The lower orders produced in high order harmonic generation can be efficiently temporally separated into monochromatic pulses by propagation in a fluoride window while still preserving their femtosecond pulse duration. We present calculations for $\mathrm{MgF}_{2}, \mathrm{CaF}_{2}$, and $\mathrm{LiF}$ windows for the third, fifth, and seventh harmonics of $800 \mathrm{~nm}$. We demonstrate the use of this simple and inexpensive technique in a femtosecond pump/probe experiment using the fifth harmonic. (C) 2008 Optical Society of America

OCIS codes: 320.0320120 .4530
\end{abstract}

High order generation (HHG) has rapidly moved from being an experiment in itself to being a tool for ultrafast science. Many groups are now using HHG, combined with long wavelength $(266-800 \mathrm{~nm})$ pulses for femtosecond spectroscopy on atoms [1] , molecules [2], and solids [3]. The spectrum of odd harmonics produced by intense femtosecond lasers in gas targets is typically divided into a "plateau" regime, where the harmonic yield is comparable from one harmonic to the next, and a "cutoff" regime, where the harmonic yield falls off rapidly with photon energy. Neglected in this description are the first few orders $(3,5,7)$ which are usually much more intense than the plateau harmonics. As demonstrated by Kosma et. al. [4] these lower orders can serve as an ideal pump pulse for many small molecules of intense theoretical interest with their first absorption band in the VUV. They also can serve as a compact table top source for angle resolved photoemission spectroscopy (ARPES) [5]. Here we demonstrate that by inserting a fluoride window into the path of an HHG beamline, the low orders of an HHG comb can be temporally separated and be used for femtosecond pump probe experiments. We emphasize that this method can be easily implemented on many existing systems.

We performed calculations for $\mathrm{MgF}_{2}, \mathrm{LiF}$, and $\mathrm{CaF}_{2}$. Table 1 lists the index of refraction $n$, group delay $\left(k^{\prime}=\right.$ $\left.n_{g} / c\right)$, and second order dispersion term $k^{\prime \prime}=d^{2} k / d \omega^{2}$ for the $3 \mathrm{rd}, 5 \mathrm{th}$, and 7 th harmonics of the ubiquitous 800 $\mathrm{nm}$ Ti:Sapphire laser. The index of refraction data and fits of LaPorte et. al. [6] were used for $\mathrm{MgF}_{2}$ and $\mathrm{LiF}$. Data of Daimon and Masumura [7] was used for $\mathrm{CaF}_{2}$. The group velocity dispersion (GVD) of the window temporally separates the harmonics. GVD also effects the pulse length, as demonstrated for LiF by Sekikawa et. al. [8]. For a chirped Gaussian input pulse with full width at half maximum (FWHM) $\tau_{0}$, and initial chirp $\beta$, de- scribed by

$$
E(t)=E_{0} \exp \left[-2 \ln 2\left(\frac{t}{\tau}\right)^{2}+i \beta t^{2}\right] \exp (i \omega t),
$$

the pulse length (FWHM) after propagating through a window of thickness $\mathrm{L}$ is given by

$$
\tau(L)=\tau_{0}\left[\left(1+2 k^{\prime \prime} L \beta\right)^{2}+\left(\frac{4 \ln (2) k^{\prime \prime} L}{\tau_{0}^{2}}\right)^{2}\right]^{1 / 2} .
$$

We present here results for $\mathrm{MgF}_{2}$. Figure 1 shows the calculated output pulselength as function of window thickness for several input pulses in the transform limited case $(\beta=0)$ and $\beta=-3 \times 10^{27} \mathrm{~s}^{2} / \mathrm{rad}$, predicted by the expression of Sekikawa et al [8] for a fundamental driver intensity of $3 \times 10^{14} \mathrm{~W} / \mathrm{cm}^{2}$. For window thicknesses of a few hundred microns, the normal dispersion of the group velocity temporally separates the 5 th and 3rd harmonics by hundreds of femtoseconds, while the

\begin{tabular}{c|c|c|c|c} 
& $\omega / \omega_{0}$ & $n$ & $k^{\prime}[\mathrm{fs} / \mu \mathrm{m}]$ & $k^{\prime \prime}\left[\mathrm{fs}^{2} / \mu \mathrm{m}\right]$ \\
\hline \multirow{3}{*}{$\mathrm{MgF}_{2}$} & $1(800 \mathrm{~nm})$ & 1.375 & 4.60 & 0.023 \\
& $3(267 \mathrm{~nm})$ & 1.398 & 4.85 & 0.092 \\
& $5(160 \mathrm{~nm})$ & 1.461 & 5.74 & 0.370 \\
\hline \multirow{3}{*}{$\mathrm{LiF}$} & 1 & 1.389 & 4.65 & 0.026 \\
& 3 & 1.415 & 4.93 & 0.101 \\
& 5 & 1.484 & 5.83 & 0.339 \\
& 7 & 1.705 & 11.32 & 3.78 \\
\hline \multirow{2}{*}{$\mathrm{CaF}_{2}$} & 3 & 1.430 & 4.79 & 0.032 \\
& 5 & 1.462 & 5.13 & 0.125 \\
& 1.553 & 6.47 & 0.593
\end{tabular}

Table 1. Index of refraction, group delay, and group delay dispersion, for the harmonics of $800 \mathrm{~nm}$ in Fluoride windows. $\mathrm{MgF}_{2}$ results are for the ordinary index 


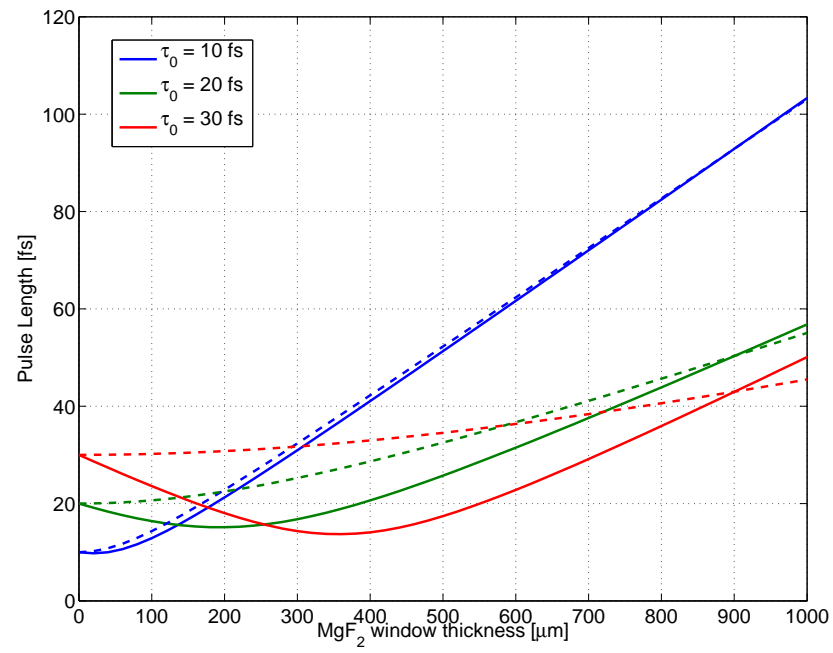

Fig. 1. Pulselength vs. propagation distance in $\mathrm{MgF}_{2}$ (ordinary index). Solid curves for chirped input pulses with $\beta=-3 \times 10^{27} \mathrm{rad} / \mathrm{s}^{2}$, dashed curves for transform limited input pulses $\beta=0$.

effect of GVD on the pulslength is minor for pulses of 20 fs or longer.

We implemented the temporal separation technique with a thick $(2.1 \mathrm{~mm}) \mathrm{MgF}_{2}$ window on a high order harmonics beamline to conduct a $162 \mathrm{~nm}$ pump, $810 \mathrm{~nm}$ probe experiment on gas phase ethylene. High order harmonics were generated by gently focusing $(\mathrm{f}=4 \mathrm{~m}) 20$ $\mathrm{mJ}, 35 \mathrm{fs}$ laser pulses into a $5 \mathrm{~cm}$ long gas cell with laser drilled pinholes, filled with 4 Torr of Xe. We estimate the fundamental intensity at focus to be $3 \times 10^{14} \mathrm{~W} / \mathrm{cm}^{2}$. The harmonic and fundamental beams were then allowed to diverge for $3 \mathrm{~m}$ where they were incident on a pair of superpolished Si Brewster angle beamsplitters [9] which remove the fundamental and reflect the harmonics. With the fundamental removed, the harmonic pulses are transmitted through the $2.1 \mathrm{~mm}$ thick $\mathrm{MgF}_{2}$ window with the c-axis oriented parallel to the propagation vector of the beam. We use a turning mirror with a $2 \mathrm{~mm}$ hole drilled 45 degrees to the surface normal to combine the VUV beam with $80 \mathrm{fs} 810 \mathrm{~nm}$ probe pulses derived from the same laser system. The VUV pulses are transmitted through the hole while the IR pulses are reflected with the central portion of the beam missing. The now colinear beams are both reflected by an $\mathrm{R}=20 \mathrm{~cm}$ metallic coated spherical concave mirror into an ethylene molecular beam. Ions produced in the focal region are selected by a $2 \mathrm{~mm}$ aperture and directed to a time of flight mass spectrometer.

Figure 2 shows the $\mathrm{C}_{2} \mathrm{H}_{4}^{+}$ion yield vs. pump/probe delay and theory based on the values of table 1 . We found the IR/IR time zero by rotating the polarization of the fundamental so that some NIR light passes through

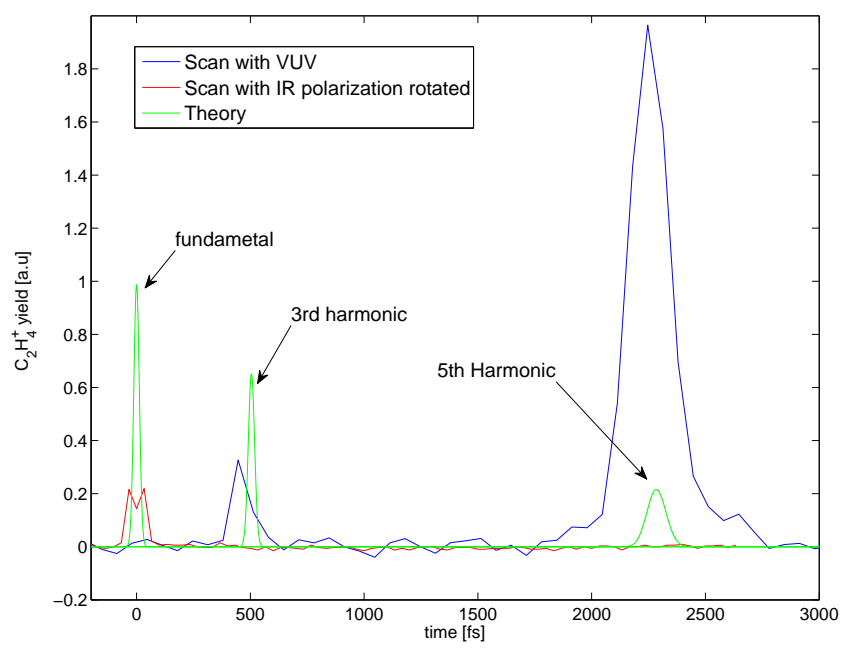

Fig. 2. Transient multiphoton ionization signals produced by the the cross correlation of the harmonic beam after propagating through a $2.1 \mathrm{~mm}$ thick $\mathrm{MgF}_{2}$ window and an $810 \mathrm{~nm}$ probe beam.

the SI beamsplitters. The positions of the transient multiphoton ionization signals agree well with theory. The signal at the position of the fifth harmonic is much larger than that at third due to the fifth harmonic being resonant with the $\mathrm{V}$ state $(\pi \pi *)$ state of ethylene.

Figure 3 shows higher resolution data around the time delay of the fifth harmonic. The zero of the time axis was determined by the multiphoton ionization of an $\mathrm{Ar}$ target. The ion yields of $\mathrm{C}_{2} \mathrm{H}_{4}^{+}, \mathrm{C}_{2} \mathrm{H}_{2}^{+}$, and $\mathrm{C}_{2} \mathrm{H}_{3}^{+}$(not shown) peak at later time delays due to nuclear motion and internal conversion in the molecule $[4,10]$. Note that although the third harmonic at 267 is also incident on the molecular target 1.7 ps earlier, since it is below the first absorption band, it does not pump the system and has no effect. This will be true for any "solar blind" system. The third harmonic would not be negligible, however, if it were temporally coincident with the fifth harmonic, as it would contribute strongly to the multiphoton ionization signal.

Since the molecular dynamics time constants [4] are much shorter than the instrument resolution, the width of the transient signals is dominated by the (chirped) VUV pulse length. By fitting the $\mathrm{C}_{2} \mathrm{H}_{4}^{+}$peak with a Gaussian, we get a FWHM of the raw signal of $157 \mathrm{fs}$. Since the energy of two additional $810 \mathrm{~nm}$ photons is needed in addition to the energy of the pump photon at $162 \mathrm{~nm}$ to ionize ethylene, we deconvolve the contribution of the $80 \mathrm{fs}, 810 \mathrm{~nm}$ probe pulse assuming the signal is proportional to the square of the $810 \mathrm{~nm}$ intensity to get a $162 \mathrm{~nm}$ pulse length of $146 \pm 10 \mathrm{fs}$.

Our results place constraints on the initial pulse length and chirp of the fifth harmonic produced in our HHG 


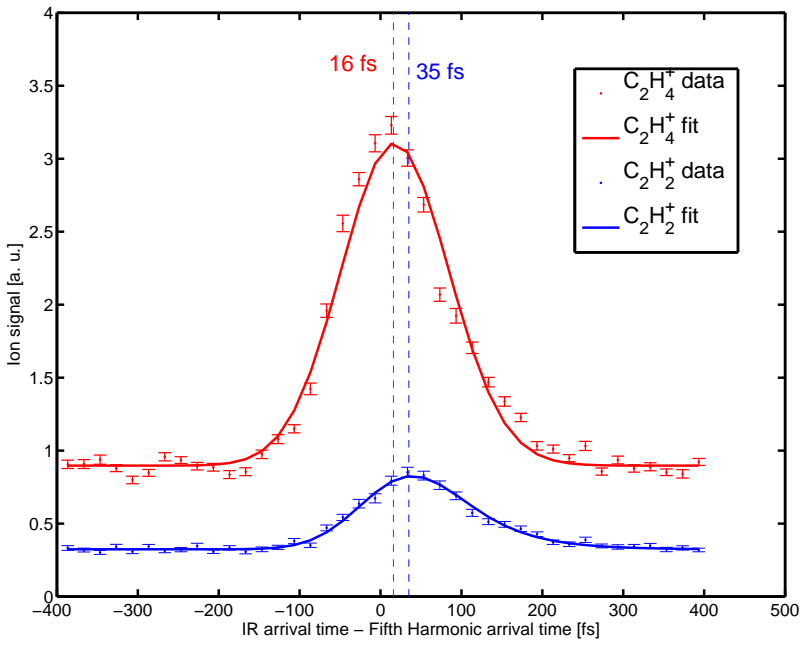

Fig. 3. Ion yields for $\mathrm{C}_{2} \mathrm{H}_{4}^{+}$and $\mathrm{C}_{2} \mathrm{H}_{2}^{+}$vs. delay between $800 \mathrm{~nm}$ probe pulse and $162 \mathrm{~nm}$ pump generated with Xe.

setup. Since perturbative nonlinear optics does not apply when generating harmonics in the strong field regime, we cannot simply estimate the pulse length at the gas cell as the fundamental pulse length divided by $\sqrt{5}$ [11]. Thus equation 2 contains two unknowns, the pulse length before the window $\tau_{0}$, and the initial chirp parameter $\beta$. We find the shortest initial $\tau_{0}$ potentially consistent with our data is $16 \mathrm{fs}$. Assuming the largest possible $\tau_{0}=35$ fs yields an intrinsic chirp parameter $\beta=-3.1 \times 10^{27}$ $\mathrm{rad} / \mathrm{s}^{2}$.

Several groups have shown that atomic dipole phase of high harmonics depends sensitively on the intensity and chirp of the fundamental pulse, and that optics with positive dispersion can be used for compression [12-14]. Here, we have also shown that dispersion can also be used as a simple way to separate harmonics for use in experiments. The technique is easy to implement in many existing systems. For example, temporally seperating the low orders in one arm of a split beam interferometer of the type described in [15], [16] can be used to conduct VUV pump/EUV probe experiments without building a separate beamline to generate each.

This work was supported by the US Dept. of Energy Office of Basic Energy Sciences, under contracts \#DEAC02-05CH1123 and \#DE-FG52-06NA26212.

\section{References}

1. M. Drescher, M. Hentschel, R. Kienberger, M. Uiberacker, V. Yakovlev, A. Scrinzi, T. Westerwalbesloh, U. Kleineberg, U. Heinzmann, and F. Krausz, "Timeresolved atomic inner-shell spectroscopy," Nature 419, 803-807 (2002).

2. E. Gagnon, P. Ranitovic, X.-M. Tong, C. L. Cocke,
M. M. Murnane, H. C. Kapteyn, and A. S. Sandhu, "Soft X-ray-driven femtosecond molecular dynamics," SCIENCE 317, 1374-1378 (2007).

3. E. Seres and C. Spielmann, "Ultrafast soft x-ray absorption spectroscopy with sub-20-fs resolution," Applied Physics Letters 91, 121919 (2007).

4. K. Kosma, S. A. Trushin, W. Fuss, and W. E. Schmid, "Ultrafast dynamics and coherent oscillations in ethylene and ethylene-d4 excited at $162 \mathrm{~nm}$," Journal of Physical Chemistry A 112, 7514-7529 (2008).

5. J. D. Koralek, J. F. Douglas, N. C. Plumb, J. D. Griffith, S. T. Cundiff, H. C. Kapteyn, M. M. Murnane, and D. S. Dessau, "Experimental setup for low-energy laser based angle resolved photoemission spectroscopy," Review of Scientific Instruments 78, 053905 (2007).

6. P. LaPorte, J. L. Subtil, M. Courbon, M. Bon, and L. Vincent, "Vacuum-ultraviolet refractive index of lif and mgf2 in the temperature range 80-300 k," J. Opt. Soc. Am. 73, 1062 (1983).

7. M. Daimon and A. Masumura, "High-accuracy measurements of the refractive index and its temperature coefficient of calcium fluoride in a wide wavelength range from 138 to 2326 nm," Appl. Opt. 41, 5275-5281 (2002).

8. T. Sekikawa, T. Katsura, S. Miura, and S. Watanabe, "Measurement of the intensity-dependent atomic dipole phase of a high harmonic by frequency-resolved optical gating," Phys. Rev. Lett. 88, 193902 (2002).

9. E. Takahashi, H. Hasegawa, Y. Nabekawa, and K. Midorikawa, "High-throughput, high-damage-threshold broadband beam splitter for high-order harmonics in the extreme-ultraviolet region," OPTICS LETTERS 29 507-509 (2004).

10. V. Stert, H. Lippert, H.-H. Ritze, and W. Radloff, "Femtosecond time-resolved dynamics of the electronically excited ethylene molecule," Chemical Physics Letters 388, 144-149 (2004).

11. T. E. Glover, A. H. Chin, and R. W. Shoenlein, "High harmonic pulse broadening in an ionizing medium," Phys. Rev. A 63, 023403 (2001).

12. J. Mauritsson, P. Johnsson, R. López-Martens, K. Varjú, W. Kornelis, J. Biegert, U. Keller, M. B. Gaarde, K. J. Schafer, and A. L'Huillier, "Measurement and control of the frequency chirp rate of high-order harmonic pulses," Phys. Rev. A 70, 021801 (2004).

13. E. Gustafsson, T. Ruchon, M. Swoboda, T. Remetter, E. Pourtal, R. López-Martens, P. Balcou, and A. L'Huillier, "Broadband attosecond pulse shaping," Opt. Lett. 32, 1353-1355 (2007).

14. T. Sekikawa, T. Ohno, T. Yamazaki, Y. Nabekawa, and S. Watanabe, "Pulse compression of a high-order harmonic by compensating the atomic dipole phase," Phys. Rev. Lett 83, 2564 (1999).

15. P. Tzallas, D. Charalambidis, N. A. Papadogiannis, K. Witte, and G. D. Tsakiris, "Direct observation of attosecond light bunching," Nature 426, 267 (2003).

16. T. Okino, K. Yamanouchi, T. Shimizu, K. Furusawa, H. Hasegawa, Y. Nabekawa, and K. Midorikawa, "Attosecond molecular coulomb explosion," Chemical Physics Letters 432, 68 (2006). 